\title{
Inappropriate Ambulance Use: A Qualitative Study of Paramedics' Views
}

\section{Utilisation inadéquate de l'ambulance : étude qualitative du point de vue des ambulanciers}

\author{
(3) \\ DEIRDRE DEJEAN, PhD \\ Postdoctoral Fellow, Department of Clinical Epidemiology and Biostatistics \\ McMaster University \\ Hamilton, ON \\ MITA GIACOMINI, PHD \\ Professor, Department of Clinical Epidemiology and Biostatistics \\ McMaster University \\ Hamilton, ON \\ MiCHELlE WELSFORD, MD, FACEP, FRCPC \\ Associate Professor, Division of Emergency Medicine \\ McMaster University \\ Hamilton, ON \\ LISA SCHWARTZ, PHD \\ Professor, Department of Clinical Epidemiology and Biostatistics \\ McMaster University \\ Hamilton, ON \\ PHILIP DECICCA, PHD \\ Associate Professor, Department of Economics \\ McMaster University \\ Hamilton, ON
}




\begin{abstract}
Introduction: Existing studies of inappropriate ambulance use focus on its extent, employing clinical criteria. Little is known about how front-line paramedics assess appropriateness. This study investigates how paramedics view and judge appropriate versus inappropriate ambulance use. Methods: We conducted interviews with 19 paramedics working in two regions in southwestern Ontario that were analyzed using grounded theory methods.

Findings: While blatantly "inappropriate" use is extraordinary, "misuse" is more common, and paramedics determine misuse largely by interpreting patients' abilities to cope with their situations. Paramedics assess this using multiple patient attributes: patient's age, knowledge of the system, system failures, social support available, presence of transportation alternatives, patient's ability to walk and trial of treatment with home remedies.

Conclusion: In the future, paramedic-informed, contextual and non-clinical criteria might supplement clinically based criteria for emergency service-use evaluation and may inform more patient-centred policy interventions to reduce ambulance misuse and inappropriate use.
\end{abstract}

\title{
Résumé
}

Introduction: Les études actuelles sur l'utilisation inadéquate de l'ambulance se concentrent sur son étendue, en employant des critères cliniques. On connaît peu la façon dont les ambulanciers de première ligne en évaluent le caractère approprié. Cette étude se penche sur la façon dont les ambulanciers perçoivent et jugent le caractère approprié, ou non, de l'utilisation de l'ambulance.

Méthodes : Nous avons interviewé 19 ambulanciers qui travaillent dans deux régions du sud-ouest ontarien. Les entrevues ont été analysées au moyen de méthodes théoriquement probantes.

Résultats: Bien qu'une utilisation « inadéquate » flagrante relève de l'exception, le « mauvais usage » est plus fréquent. Les ambulanciers définissent habituellement un « mauvais usage » en fonction des capacités du patient de prendre en main sa situation. Pour ce faire, ils emploient plusieurs caractéristiques : l'âge du patient, sa connaissance du système, les failles du système, la disponibilité d'un soutien social, la présence d'autres moyens de transport, la mobilité du patient et l'utilisation de remèdes maison.

Conclusion: À l'avenir, des critères éclairés par les ambulanciers, contextuels et non cliniques pourraient supplanter les critères cliniques pour l'évaluation de l'utilisation des services d'urgence et pourraient éclairer des politiques d'intervention davantage centrées sur le patient afin de réduire le mauvais usage et l'usage inadéquat de l'ambulance. 
When non-urgent patients are triaged to lower emergency department (ED) priority, they may wait many hours for care. If they arrived by ambulance, the paramedics may need to wait with them, delaying response to other emergency calls, and depriving the paramedics of opportunities for meals, sleep and education (Marks et al. 2002; Pennycook et al. 1991). The inappropriate use of EMS drains health system resources and undermines paramedic morale (Palazzo et al. 1998; Ting and Chang 2006; Zachariah 1999). For non-urgent patients, ED care may be less effective than primary care, and can ultimately delay their appropriate care (Drummond 2002; Gill 1994).

Health services research on ambulance usage, to date, has relied largely on clinical definitions and criteria for appropriateness. The extent of inappropriate ambulance use ranges from 11.3\% (Billittier et al. 1996) to 51.7\% (Morris and Cross 1980) of calls; most estimates approximate 30\%. Most studies rely on the judgement of the ED physician (Billittier et al. 1996; Camasso-Richardson et al. 1997; Gardner 1990; Little and Barton 1998; Palazzo et al. 1998) or attending nurse (Frank and de Villiers 1995) to assess appropriateness. Only two studies have involved the perspective of paramedics, but these used pre-specified (Hjalte et al. 2007) or unspecified (Richards and Ferrall 1999) indicators of inappropriateness. No study has developed a definition or measure of inappropriate ambulance use that draws on the expertise and experiences of paramedics.

The Ontario ambulance billing policy presents an interesting context for the study of the meaning of inappropriate ambulance use. In Ontario, medically necessary ambulance transports are funded under the Ontario Health Insurance Plan, with patients normally being responsible for a $\$ 45$ co-payment. Some users are exempt from the co-payment, including those receiving social assistance, living in a long-term care facility or home for special care or being transferred from one health facility to another. If the attending ED physician deems the ambulance use to have been medically non-essential, patients are billed $\$ 240$ for the ambulance service (MOHLTC 2015). The hospitals bill for and collect the fees; $\$ 15$ is remitted to the province for each type of call, while the remainder is kept by the billing hospital (MOHLTC 1992).

For health system evaluation and quality improvement, reducing inappropriate emergency service use requires meaningful definitions and measures that reflect valid professional, patient and societal perspectives on why ambulance care and transport might be needed, as well as misused. This study focuses on the typical first steps of the patient's journey to the ED - the ambulance call and transport - and provides insights into how paramedics' perceptions of appropriate versus inappropriate cases might differ from existing conceptualizations. Paramedic perspectives provide especially rich insights into underappreciated non-clinical and contextual factors that both heighten emergencies for patients and legitimize them in the eyes of paramedics. We present a theory of inappropriate ambulance use according to paramedics' perceptions, which could be useful to improve future evaluations of EMS use and interventions to control inappropriate use. 


\section{Methods}

Study design

We used constructivist grounded theory methodology (Charmaz 2006). This inductive and exploratory method is well-suited for pursuing new insights without imposing pre-existing constructs (i.e., about the nature of inappropriate ambulance use). This study received research ethics approval from the Hamilton Integrated Research Ethics Board (\#10-373).

\section{Study sample and recruitment}

Paramedics were recruited from two regions in southwestern Ontario that include urban municipalities and rural townships, each with a population of approximately 500,000. Paramedics were invited to participate in the study through e-mail invitations. The only inclusion criterion was that the participant had to have worked as a paramedic responding to patient calls within the last year. Within each region, several paramedics initially responded to the e-mail, and additional participants were recruited through snowball sampling (Patton 2002). New participants were recruited and interviews continued until theoretical saturation (Charmaz 2006).

\section{Data collection}

Semi-structured telephone interviews were conducted between March 2011 and June 2012 with 19 paramedics. Interviews lasted between 27 and 64 minutes (average 38 minutes) and were audio-recorded and transcribed verbatim. Each interview involved open-ended questions about the role of paramedics in the healthcare system, perceptions of appropriate ambulance use, beliefs about ambulance billing policies and factors believed to contribute to inappropriate use.

\section{Data analysis}

Data were gathered from interviews, transcribed verbatim and coded in stages to build a theory of inappropriate use from the paramedic perspective (Charmaz 2006). This paper reports the aspect of the theory addressing coping, specifically. Interviews were conducted and analyzed in an iterative process, according to grounded theory conventions (Charmaz 2006). One author coded all transcripts; a second author analyzed a sample of transcripts independently to compare initial coding categories. Differences were resolved by consensus.

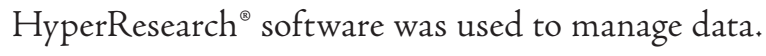

\section{Findings}

\section{Appropriate and inappropriate uses}

Perhaps not surprisingly, notions of the appropriate use of ambulances tend to be focused most prominently on the typical "lights and sirens" emergency responses. Examples of appropriate ambulance use shared by paramedics were often the most obvious life-threatening cases such as heart attacks, strokes, motor vehicle collisions or severe allergic reactions. 
Although all respondents agreed that these situations justify emergency response, there was less agreement about non-emergent situations. Paramedics noted that the appropriateness of a call can be quite subjective and "varies from person to person" (P\#4). The variability of appropriateness is partially due to personal definitions and experiences of an emergency. Many paramedics acknowledged that appropriateness is a subjective concept and that patients might experience "personal emergencies" (P\#16), even if they do not fit within the more objective understandings of an "emergency."

Comparable to the cited examples of appropriate use, the most prominent examples of inappropriate use described by paramedics were often the most extreme. We characterize these unambiguous situations of inappropriate use as abuse of the system. The concept of abuse refers to patients who knowingly manipulate the system, particularly those who feign medical emergencies simply for transportation to a destination near the hospital.

There are frequent flyers who deliberately abuse the system for a transport. Just to get into an area that they want to go to. That is a willful and clearly, I mean, they know. They've been informed, either by the EMS system or the paramedics themselves, that this is a manipulation and it's inappropriate, and they continue to do so. (P\#12)

The misuse of ambulance services is much harder to define, and often arises because of external circumstances or genuine misunderstandings about how the ambulance system works. While the extreme examples of appropriate use and abuse engendered universal agreement about classifications, the more moderate examples of ambulance misuse elicited more nuanced definitions of appropriateness.

\section{Distinguishing inappropriate use through perceived coping}

Paramedics relied on their perception of the patients' ability to cope with the situation that provoked the call to 911 to determine the appropriateness of ambulance use. Recognition of the patient's (lack of) ability to cope is typified by the following paramedic's reaction to calls for minor (and potentially inappropriate) issues:

So a lot of times we get really minor calls for things, and although to us it seems like, oh my god, what a waste of our time, that I didn't go to school for all this time and whatever, and I don't have all this equipment on my truck to, like, pat you on the back and tell you that it's okay, and, you know, be your taxi essentially. But we do that all the time. But it's because to that person, they've reached their limits of coping. (P\#3)

Paramedics cited four perceived factors that contribute to the patients' inability to cope with their situations and that might justify the use of the ambulance: the patient's age, lack of knowledge of the system, system failures and lack of social support. 


\section{Patient's age}

Some paramedics suggested that the appropriateness of an ambulance call could vary depending on the age of the patient, even for the same medical condition. An example of this is the occurrence of respiratory illness:

[I]t really is particular to certain age groups. You've got your 90-year-olds who have pneumonia that, you know, they can't move and when they get pneumonia, they don't have enough people with them, they don't have family or they don't have - you know, they need to go to the hospital, they need to get treatment. Now you bump that down into your 30-, 40-, 50-year-olds that, you know, are coughing up green phlegm and they have a fever, and their family is all around when we get there, I mean, you don't need us. Yes, I understand that you're feeling ill, but you don't need to call 911. (P\#13)

Paramedics noted that age often affected their perception of the appropriateness of the call because it contributes to the patient's ability to physically cope with illness. In the example above, the effects of respiratory illness left untreated might be much more dire in an elderly patient than in a healthy adult. Similarly, paramedics considered the presence of a fever to be more serious in the paediatric population than in the adult population, and more likely to warrant emergency response.

\section{Lack of knowledge of the system}

Patients sometimes misuse the ambulance system because they are experiencing a health or social need, but are unsure of how to seek help. Paramedics described being called simply to offer reassurance to patients or to act as "information providers" (P\#4) who could direct the patient to more appropriate resources. Even when patients are aware that the ED might not be the appropriate course, if their 911 call stems from uncertainty about the system and a genuine need, it is more likely to be considered appropriate.

A lot of times, people don't necessarily need to go to the hospital, but they're not sure what to do. Or their family's not sure what to do with them. And so we end up filling that gap in the healthcare system, you know, maybe helping them to facilitate CCAC [Community Care Access Centre] or other homecare services. (P\#3)

\section{System failures}

While patients sometimes misuse ambulance services because they are unaware of the alternatives available to them, other times they have tried and have been failed by those alternatives. Paramedics are more likely to consider these cases appropriate when caused by gaps in the healthcare system. In particular, the paramedics interviewed consistently noted the number of individuals left waiting for placement in a long-term care facility. 
The needs of patients living at home because they are unable to secure more appropriate care environments can be quite draining on the ambulance system, but they are seen as legitimate needs because the patients are unable to cope in the home setting.

But there's kind of a huge gap right now in healthcare where people will ... you know, they're not coping well at home anymore and they're waiting for placement into a long-term care facility or an assisted living facility. And until they have a tragic accident like they fall and break their hips, they don't really qualify. Like they're so far down on the waiting list that they're waiting for years for placement. (P\#3)

Paramedics also expressed sympathy for patients who entered the healthcare system via the ambulance and ED, but whose medical needs were not appropriately addressed. The most notable examples of these situations were patients with mental health issues, who often present to the ED but, from the perspective of paramedics, are not given adequate support when discharged from the hospital:

[T] hey are in crisis a lot of the time. Even if it is kind of daily and painful. So, I don't think it's so much saying it's non-essential, I think maybe the system is failing them somewhere else and that's why they're getting out and they're calling because they need to be back and back and back. They're not getting any answers to their problems, that's why they keep coming back to us. So I don't know if I would call them non-essential ... (P\#7)

The emergency system itself can also fail patients when wait times feel unbearable. The quintessential example of an inappropriate use of ambulance services is when the patient has already been transported to the hospital and calls 911 from the waiting room in the hopes of being seen more quickly. While calling from the hospital can be considered a clear abuse of the ambulance system, if paramedics recognize that it is caused by a failure of the system, it can be seen as an understandable misuse rather than blatant abuse. Indeed, paramedics perceive travelling to the ED as an attempt to cope with the situation, and if the wait for care lengthens, the patient's ability to cope may subsequently change. One paramedic, who expressed sympathy for a call placed from the hospital waiting room, explained this concept:

We can give them something, they're not sitting on a chair, they're on a bed. If it's a fracture, we'll mobilize and they're given pain relief, depending on the service they called, some services can't give pain relief. So, yeah, that I could understand. Yes, it would be appropriate for you to go in the door and get treated but I can understand how that avenue hasn't worked too well for you, so you're trying something else. (P\#16) 


\section{Lack of social support}

Finally, the existence or presence of other social support might contribute to the patient's ability to cope with their situation. For example, the appropriateness of lift assists depends on whether the patient has family or friends who might help instead:

I know some medics get upset about [lift assists], but the way I see it, well I mean, you can't just ... like, say someone has a fall and they have no family in the area. They kind of fall through the cracks of who's going to help them get up, right? (P\#10)

In the absence of formal or informal caregivers to assist the patient, lift assists are perceived as appropriate uses of the ambulance system. In other cases, patients may have support, but caregiver limitations push them to unnecessarily use the ambulance because they are unable to safely transport the patient. For example, an elderly woman might be too frail to assist her spouse to the car and drive to the doctor's office.

In some cases, the patient and the family don't actually want to go to the ER, if they need some assistance to get somewhere but they themselves realize that they're not needing to go to the ER, but that's the only option that we're able to provide, therefore that's what we provide. Again, it's not somebody who's abusing the system or making inappropriate calls. It's somebody who legitimately needs some level of assistance but not necessarily direct medical assistance at that time, and definitely not the time and the resources of the ER. (P\#17)

Although the use of the ambulance (and the ED, subsequently) is traditionally considered inappropriate in these situations, paramedics perceived it as appropriate because they recognized that the families and patients had no other way of coping with a legitimate medical need. They noted that one of the gaps filled by paramedicine is the absence of the traditional house call; patients who are unable to leave the house rely on the emergency system for both urgent and non-urgent needs.

[W]e're really the only profession that provides any house calls anymore, you know when these doctors used to go and they'd have a group of patients and they'd provide house calls. Well there's very few family physicians that provide house calls, $[\ldots]$ really we're the only ones that if you call, we will come. And we will provide you with care at home, wherever your home may be. So, it's kind of one of those, you know, that safety net. (P\#8)

While the aforementioned factors contribute to paramedics' perceptions of appropriate ambulance use, other cues highlight the patients' inadequate attempts to cope with their situation. In particular, paramedics were more likely to perceive an ambulance call 
as inappropriate if there was alternative transportation available, if the patient was ambulatory or if the patient did not attempt any home remedies.

\section{Alternative transportation available}

Many paramedics, when asked to describe inappropriate use, remarked on the presence of "cars in the driveway" as a sign that the patient might be misusing their services. In particular, they expressed frustration when the family followed them to the hospital in a private vehicle.

Um, you know, let's say "I pulled a muscle in my back" say, yesterday, they call us today and they walk to the vehicle and we bypass two or three cars sitting in the driveway. I've picked up patients where they shovel the sidewalk for us to walk a patient to the vehicle with a back injury, and they were warming up a car to follow us to the hospital. And this patient sat in our vehicle. I remember this call precisely, it was the middle of January, two in the morning, car was running and they were defrosting their car. But you couldn't say to them "why wouldn't you just take this person in the vehicle with you?", that was not my job, my job is to bring them in. (P\#11)

\section{Ambulatory}

The presence of alternative transportation alone is not a sufficient marker of inappropriate use; paramedics' perceptions of inappropriateness are exacerbated when the patient is also able to walk to the ambulance. Paramedics take the ability to walk as a sign that the patients might have been able to transport themselves to the hospital or to another care destination:

Especially the ambulatory patients. Like, people that we honestly, we don't even have to get out the stretcher for them because they meet us at the end of their driveway or they meet us at the front door, right? I mean, the way I see it personally is if we're not giving you a drug and you're able to walk yourself, well, I mean there's really no reason for you to be taking an ambulance then. $(\mathrm{P} \# 10)$

Other paramedics, despite being frustrated by patients who appear to be able to transport themselves to the hospital, acknowledged that transportation of patients is part of their role, as one explained, "Yeah, I think whether we like it or not, sometimes we are just a bus or a taxi service, I think that is reality, and transportation is part of it." (P\#6)

\section{No attempt at home remedies}

Paramedics expressed frustration when it did not appear that patients had attempted to care for themselves at home before calling the ambulance system. They cited the flu as an example of a medical condition that could be considered an appropriate use of the ambulance if it progressed to the point where the patient could no longer cope with the symptoms. 
If the patient had not attempted to cope using common home remedies, the use was more likely to be considered inappropriate. The perception of inappropriateness stems from the notion that the patient should have known how to cope with the situation, which is highlighted in the following passage that juxtaposes two potential responses to flu-like symptoms:

Even when it comes down - as an example, somebody with the flu. I had a retired nurse who was like 57 years old, otherwise healthy who had had nausea, vomiting and diarrhea for like two hours. She hadn't done anything to help herself and now was calling an ambulance to go to the emergency department, where somebody else who has been sick for two and a half days and has been trying fluids and Gravol and Tylenol and all the stuff that you are supposed to do and staying home and trying to run it out, and is now not coping and is severely dehydrated and needs some help, then no problem, even if you are twenty-five and perfectly healthy. If you have done what you are supposed to do and it hasn't worked out and now you need help, then that is what we are here for, right? But when you know better and you haven't bothered to try to help yourself, it is frustrating when you now inappropriately deplete resources because you couldn't help yourself, for no good reason other than you haven't bothered. (P\#5)

Although the causative medical condition described is identical, the patients' attempts and ability to cope with the situation before accessing 911 clearly swayed paramedics' perceptions of the appropriateness of ambulance use.

Taken together, these cues contribute to the paramedics' perception of whether the patient was able to cope with their situation and, in turn, differentiate appropriate and inappropriate ambulance uses. When there are sufficient factors that suggest that the patient was unable to cope, paramedics are likely to perceive the ambulance use as appropriate (Figure 1). Conversely, certain cues are used to judge whether the patient should have been able to cope, and lead to a perception of inappropriate use. Paramedics use these non-clinical cues to assess the patients' ability to cope, so, unlike traditional definitions of appropriateness that rely on clinical criteria, patients presenting with the same clinical symptoms could be characterized as either appropriate or inappropriate use.

\section{Discussion}

Along the continuum of inappropriate to appropriate ambulance use, the extremes can be characterized with little dispute. Paramedics typified appropriate ambulance use as those cases appropriate for "lights and sirens" responses to life-threatening emergencies, such as heart attacks, strokes and motor vehicle collisions. At the other extreme, paramedics characterized inappropriate uses in terms of the most blatant abuses, for example, patients who fabricated symptoms or exited at the ED but walked away from the hospital. These cases are extraordinary but memorable. 
FIGURE 1. Factors that contribute to paramedics' perceptions of patients' attempts and ability to cope

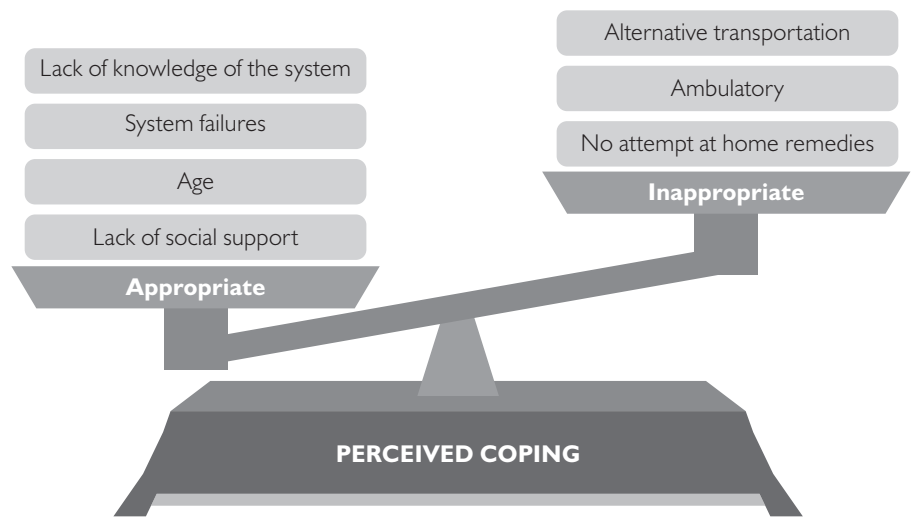

Between these indisputable opposites, however, lies a large area of ambiguity where paramedics must interpret appropriateness. Most ordinarily, inappropriate use takes the form of ambulance misuse: improper use that is unintentional or unavoidable. In this case, the health condition is not emergent, but the patient or family perceives no other way to access the healthcare system - either because of their own limitations or because of barriers to a more appropriate level of service. Misuse is challenging to identify, and requires paramedics to interpret the patient's situation holistically and beyond clinical criteria. In addition to the health condition, paramedics assess both the patient in context (e.g., age and physical environment) and whether the patient has attempted to cope with their condition and made use of the resources available to them (e.g., access to other types of healthcare, social support, alternative transportation or care). In the absence of these resources - whether because the patient has been failed by their social network or the healthcare system more broadly - paramedics are inclined to judge ambulance transport as "appropriate" for urgent but non-emergency conditions.

This study is, to the best of our knowledge, the first in-depth analysis of paramedics' perceptions of inappropriate ambulance use. It gives voice to those at the front-lines of ambulance services, who interpret appropriateness of use in patient-centred contexts. It is interesting that, when talking about misuse and inappropriate use, paramedic participants in this study often lapsed from talking about patients, in general, in third person to more pointed admonishments for "you," in second person (e.g., "I don't have all this equipment on my truck to, like, pat you on the back and tell you that it's okay [...]") - sharing judgments in the context of this study that they do not voice directly to the patients they serve. These interior monologues provide important input for health service administrators and policy makers.

Our findings about paramedics' perceptions of coping complement the few studies that have examined patient and caregiver decision-making when calling for an ambulance. Patients describe their experience of the situation as "intolerable" (Ahl et al. 2006) and cite feelings of anxiety before calling (Ahl et al. 2006; Booker et al. 2013). Booker et al.s (2013) study of patients who called an ambulance for primary care problems revealed a misunderstanding of options in the healthcare system or previous negative experiences seeking care as factors influencing the decision to call, which is consistent with the cues we found that paramedics use to identify a patient's inability to cope. 
This study also builds on previous studies that have examined the perceived prevalence of inappropriate use by asking paramedics to characterize cases of appropriate and inappropriate use while presupposing the criteria that should be used to make that distinction. These empirical studies of inappropriate ambulance use have focused primarily and often solely on clinical criteria. Our examination of how paramedics view and judge appropriate versus inappropriate ambulance use found that paramedics also tend to characterize the appropriateness of ambulance use according to contextual information gathered on scene.

Ultimately, addressing the problem of ambulance misuse requires asking fundamental questions about what the role of paramedics and ambulances should be in the healthcare system. In the Ontario context, the patient initiates ambulance use, and paramedics are unable to refuse transportation or to transport patients to alternative care settings. In addition to the patient attributes noted in this study, more research is needed about the effect of organizational and systemic factors on ambulance use and perceptions of appropriateness.

Traditionally, ambulance service was designed to provide rapid transport to the hospital ED for patients experiencing a medical emergency (Shah 2006). Where the role of paramedics expands to fill other gaps in the healthcare system, conceptions of appropriateness also change in response. Policy responses could either reframe the role of ambulance services to meet the needs of those patients (e.g., through increased community paramedicine initiatives [Bigham et al. 2013]) or target those inconsistencies with mechanisms for deterrence such as user fees and education campaigns. To date, there has been no empirical evaluation of the impact of the co-payments and billing policies on appropriate use in Ontario. However, our findings suggest that paramedics' definition of inappropriate use can conflict with the traditional role, and that they recognize the needs of patients beyond the clinical criteria typically used to define appropriate use.

\section{Conclusions}

The results of this study contribute to shared understandings of appropriate and inappropriate ambulance use. Specifically, the cues that paramedics use to define and identify inappropriate use differ from the clinically focused definitions prominent in the emergency health services literature. Paramedics have unique voices and perspectives to offer health policy makers and system administrators, with their broader view of the social and organizational context within which the patient is seeking help. Their understanding of appropriate ambulance use is more holistic than the information considered in other assessments. For health services research into use patterns and inappropriate use rates, clinical diagnoses or hospital admission status may only offer a limited portrayal of the patient's experience. In the future, paramedic-informed, contextual and non-clinical criteria might supplement clinically based criteria for emergency service use evaluation, and inform more patient-centred policy interventions to reduce ambulance misuse and inappropriate use.

\section{Acknowledgements}

Deirdre DeJean acknowledges the support of a Centre for Health Economics and Policy Analysis (CHEPA) Doctoral Fellowship and a Canadian Institutes for Health Research (CIHR) CGS Doctoral Award. We thank members of the CHEPA Polinomics group for valuable comments on an earlier draft of this paper. 
Correspondence may be directed to: Deirdre DeJean, $\mathrm{PhD}$, Postdoctoral Fellow, Dept. of Clinical Epidemiology \& Biostatistics, McMaster University, CRL 202, 1280 Main St. W., Hamilton, ON L8S 4K1; tel: 905-525-9140 ext. 28604; e-mail: dejeand@mcmaster.ca.

\section{References}

Ahl, C., M. Nystrom and L. Jansson. 2006. “Making up one's Mind - Patients' Experiences of Calling An Ambulance." Accident and Emergency Nursing 14(1): 11-19.

Bigham, B., S. Kennedy, I. Drennan and L. Morrison. 2013. “Expanding Paramedic Scope of Practice in the Community: A Systematic Review of the Literature." Prehospital Emergency Care 17(3): 361-72.

Billittier, A.J., R. Moscati, D. Janicke, E.B. Lerner, J. Seymour and D. Olsson. 1996. “A Multisite Survey of Factors Contributing to Medically Unnecessary Ambulance Transports." Academic Emergency Medicine 3(11): 1046-50.

Booker, M., R. Simmonds and S. Purdy. 2013. “Patients Who Call Emergency Ambulances for Primary Care Problems: A Qualitative Study of the Decision-Making Process." Emergency Medicine Journal 31(6): 1-5. doi:10.1136/emermed-2012-202124.

Camasso-Richardson, K., J.A. Wilde and E.M. Petrack. 1997. “Medically Unnecessary Pediatric Ambulance Transports: A Medical Taxi Service?" Academic Emergency Medicine 4(12): 1137-41.

Charmaz, K. 2006. Constructing Grounded Theory: A Practical Guide Through Qualitative Analysis. London: SAGE Publications.

Drummond, A. 2002. "No Room at the Inn: Overcrowding in Ontario's Emergency Departments." CJEM 4(2): 91-97.

Frank, S.A. and P.J. de Villiers. 1995. "An Analysis of the Appropriate Use of the Caledon Ambulance Service in the Overberg: A Short Report." South African Medical Journal, Suid-Afrikaanse Tydskrif Vir Geneeskunde 85(11): 1185-86.

Gardner, G.J. 1990. "The Use and Abuse of the Emergency Ambulance Service: Some of the Factors Affecting the Decision Whether to Call An Emergency Ambulance." Archives of Emergency Medicine 7(2): 81-89.

Gill, J. 1994. "Nonurgent Use of the Emergency Department: Appropriate or Not?" Annals of Emergency Medicine 24(5): 953-57.

Hjalte, L., B.-O. Suserud, J. Herlitz and I. Karlberg. 2007. "Why Are People Without Medical Needs Transported by Ambulance? A Study of Indications for Pre-Hospital Care." European Journal of Emergency Medicine 14(3): 151-56.

Little, G.F. and D. Barton. 1998. "Inappropriate use of the Ambulance Service." European Journal of Emergency Medicine 5(3): 307-11.

Marks, P., T. Daniel, O. Afolabi, G. Spiers and J. Nguyen-Van-Tam. 2002. “Emergency (999) Calls to the Ambulance Service That Do Not Result in the Patient Being Transported to Hospital: An Epidemiological Study." Emergency Medicine Journal 19(5): 449-52.

Ministry of Health and Long-Term Care (MOHLTC). 1992. Ambulance Co-Payment Billing Manual. Toronto, ON: MOHLTC. Retrieved December 10, 2015. <http://www.ambulance-transition.com/pdf_documents/ amb_copayment_billing_manual.pdf>.

Ministry of Health and Long-Term Care (MOHLTC). 2015. "Ambulance Charges." Retrieved November 25, 2015. <www.health.gov.on.ca/english/public/program/ehs/land/service_qa.html>.

Morris, D.L. and A.B. Cross. 1980. “Is the Emergency Ambulance Service Abused?” BMJ 281(6233): 121-23.

Palazzo, F.F., O.J. Warner, M. Harron and A. Sadana. 1998. "Misuse of the London Ambulance Service:

How Much and Why?" Journal of Accident \& Emergency Medicine 15(6): 368-70.

Patton, M.Q. 2002. Qualitative Research \& Evaluation Methods (3rd ed.). Thousand Oaks, CA: SAGE Publications.

Pennycook, A.G., R.M. Makower and W.G. Morrison. 1991. "Use of the Emergency Ambulance Service to an Inner City Accident and Emergency Department-A Comparison of General Practitioner and '999' Calls." Journal of the Royal Society of Medicine 84(12): 726-27.

Richards, J. and S. Ferrall. 1999. "Inappropriate Use of Emergency Medical Transport: Comparison of Provider and Patient Perspectives." Academic Emergency Medicine 6(1): 14-20.

Shah, M.N. 2006. "The Formation of the Emergency Medical Services System." American Journal of Public Health 96(3): 414-23.

Ting, J. and A. Chang. 2006. “Path Analysis Modeling Indicates Free Transport Increases Ambulance Use for Minor Indications." Prehospital Emergency Care 10(4): 476-81.

Zachariah, B. 1999. “The Problem of Ambulance Misuse: Whose Problem Is It, Anyway?” Academic Emergency Medicine 6(1): 2-5. 